\title{
Recognition of brain cancer and cerebrospinal fluid due to the usage of different MRI image by utilizing support vector machine
}

\author{
Soobia Saeed, Afnizanfaizal Abdullah \\ Department of Software Engineering, Faculty of Computing, Universiti Teknologi Malaysia, Malaysia
}

\begin{tabular}{|c|c|}
\hline Article Info & ABSTRACT \\
\hline $\begin{array}{l}\text { Article history: } \\
\text { Received Oct 23, } 2019 \\
\text { Revised Dec 30, } 2019 \\
\text { Accepted Jan 31, } 2020\end{array}$ & $\begin{array}{l}\text { Medicinal images assume an important part in the diagnosis of tumors } \\
\text { as well as Cerebrospinal fluid (CSF) leak. Similarly, MRI could be } \\
\text { the cutting-edge regenerative imaging technology that allows for a sectional } \\
\text { angle perspective of the body that gives specialists convenience and will } \\
\text { inspect the person-concerned. In this paper, the author has attempted } \\
\text { the strategy to classify MRI images at the beginning of production to have }\end{array}$ \\
\hline $\begin{array}{l}\text { Keywords: } \\
\text { Brain tumor } \\
\text { Cerebrospinal fluid } \\
\text { Classifier } \\
\text { HOG (histogram of a gradient) } \\
\text { MRI } \\
\text { SVM }\end{array}$ & $\begin{array}{l}\text { a tumor or recognition. The study aims to address the atorementioned } \\
\text { problems associated with brain cancer with a CSF leak. This research, } \\
\text { the author focuses on brain tumor and applies the statistical model } \\
\text { for the testing and also discusses the images of a brain tumor. They can judge } \\
\text { the tumor region by conducting a comparative image analysis and applying } \\
\text { Histogram function afterwards to construct a classifier that could be prepared } \\
\text { to predict tumor and non-tumor MRI examinees based on the support vector } \\
\text { machine. Our system is capable of detecting the right region that } \\
\text { a pathologist also highlights. In the future, this should be more driven with } \\
\text { the objective that tumors can be arranged and describe the solution } \\
\text { in the medical terms \& implementation with gives some predictions about } \\
\text { the future generated by modified technology. }\end{array}$ \\
\hline
\end{tabular}

This is an open access article under the CC BY-SA license.

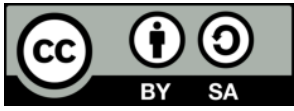

\section{Corresponding Author:}

Soobia Saeed,

Department of Software Engineering, Faculty of Computing,

Universiti Teknologi Malaysia, 81310 Skudai, Johor Bahru, Malaysia.

Email: saeed.soobia@graduate.utm.my

\section{INTRODUCTION}

Brain tumor and CSF identify with magnetic resonance images (MRI) is critical in the medicinal determination as it gives organized information on the design outline of a body part. Medical imaging is a crucial segment connected with countless which helps to conclude. The best component of MRI is, so it can create depicting images of diverse features. There is a couple of essential MRI checks out: T1 weighted MRI as well as T2 weighted MRI. T1 pictures are generally used to take a gander at typical anatomical subtle elements. T1 is best to look at the cerebrum structure because fats and tissues seem brilliant and bone marrow contains a lot of fat. T2 is the transverse development of protons and is typically used to take a gander at pathology because most tissues included in contagion tend to have higher water content than normal.

The white matter appears a light grey in T1 and a dark grey in T2. Cerebrospinal fluid (CSF) is a clear, colorless body fluid found in the brain and spinal cord. It is produced by the specialized ependymal cells in the choroid plexuses of the ventricles of the brain and absorbed in the arachnoid granulations. Figure 1 shows the indication of both features are grey matter appears grey in both and another one CSF performs black in $\mathrm{T} 1$ and white in $\mathrm{T} 2$. 


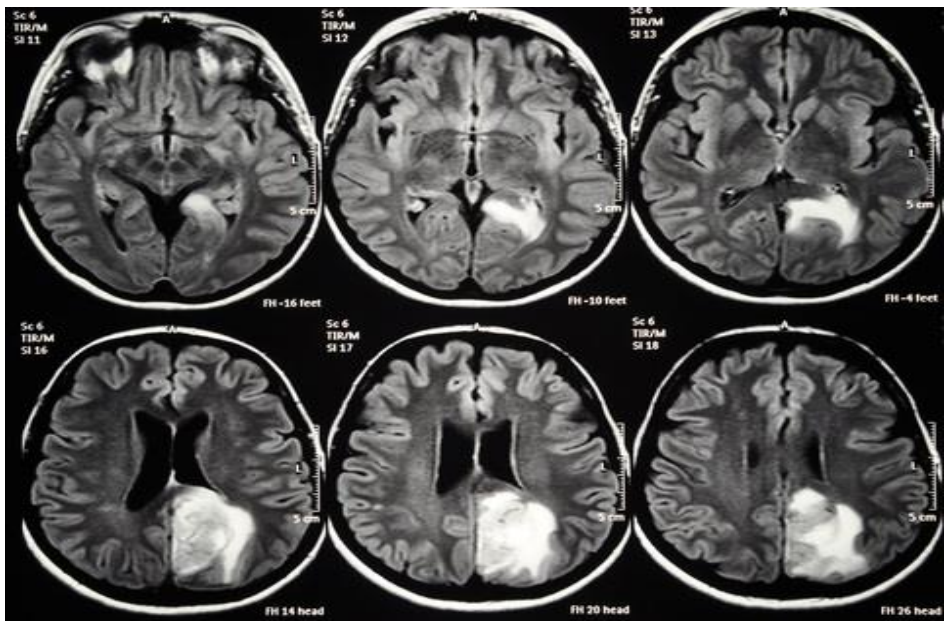

Figure 1. The above image shows the comparison between T1 weighted MRI scans and T2 weighted MRI images [1]

Figure 1 indicates the presence of cerebrospinal fluid (CSF) in T1 as black and T2 as white. The white matter appears in T1 as a light grey and in T2 as a dark grey. In both, the grey matter appears grey. Figure 1 shows the correlation among T1 weighted MRI scans and T2 weighted MRI. As we are concentrating on the tumorous area so T1 weighted MRI outputs are most useful for us as they can help us in examining the points of interest of anatomical conduct of tumorous region. So, we have utilized T1-weighted images for preparing our model. MRI is the best in medical imaging technology, which permits the cross-sectional perspective of the body with uncommon tissue contrast [2]. MRI assumes a vital part in evaluating neurotic states of the lower leg, foot and cerebrum. MRI is a non-obtrusive methodology that has ended up being as compelling instrumental in the investigation of the human cerebrum. The data that MRI gives has enormously expanded information on ordinary and diseased anatomy about medical research and is a basic part of the determination and treatment arranging [3]. The choice of medicinal medication by the physician relies on diagnostic tests, making the accuracy of the diagnosis a key factor in medical care. It is possible to measure the properties of the final tests. In the view of these properties, the most suitable test can be chosen for a given disease condition and precision are commonly used to show the effects of a test.

- Tumor detection

The human brain is the central organ of the human nervous system, and the central nervous system is created by the spinal cord. The brain is made up of the heart, brain and cerebellum. The brain is contained in the head's skull bones and protected by them. The brain is a thick, spongy tissue that is surrounded by a Skull, a rigid bone with three thin layers of tissue called meninges and aqueous fluid. The brain is responsible for all the major actions required by a human body, such as thinking, walking, talking, etc. Our senses such as sight, hearing, touch, taste, smell, memory, emotions, and personality also control it.

The detection of anomalous tissue development is essentially convinced by the need to achieve as much accuracy as possible [4]. Most cells in the body develop and then divide in an orderly manner to produce additional cells to keep the human body healthy and functioning properly. When cells lack the ability to expand or evenly divide, the extra cells produce tissue masses called tumors.

- Primary cancer

Cancers, which begin in the mind cells, are called as essential cerebrum cancers. For the situation of essential cerebrum cancers, now and again they extend to different parts of the mind or the spine. In any case, scattering to different organs happens just hard.

- Secondary cancer

An auxiliary cerebrum cancer, otherwise called a metastatic mind cancer, happens when growth cells spread to your mind from another organ, for example, your lung or bosom.

- Benign tumors

Benign tumor scan be removed and they seldom re-grow. The border or edge of a benign brain tumor can be seen. Cells from benign tumors do not invade tissues to other parts of the body but they are dangerous as well because they can press on the sensitive area of the brain and made the brain to work improperly and cause serious health issues. Very rarely a benign brain tumor may become malignant. 
- Malignant tumor

Malignant brain tumors are extreme and life-threatening in patients that may be primary (a tumor that originates exclusively from brain tissues) or secondary (metastasis that originates from another part of the body) they are likely to grow rapidly and may invade normal brain tissues around them. One of the real reasons for the rise in mortality of children and adults is a brain tumor. A tumor is a mass of tissue that, by the typical forces that direct development, becomes uncontrollable [5]. The occurrence of brain tumors is higher than ever, especially in older adults. A brain tumor with CSF is a collection of abnormal cells that develop within the brain or around the brain. Tumors can specifically crush all healthy cells of the brain. It can also affect healthy cells around the tumor and affect different parts of the brain by causing inflammation, swelling, and creating pressure within the skull $[1,6]$. Taking into account the exact inference, early detection and proper treatment are important measures to improve disease outcomes. Brain abnormalities include a wide variety of conditions ranging from developmental defects to neurological disorders. This uncertainty results in incalculable potential results from prenatal ultrasound findings that could result in some theoretical predicaments [1].

\section{LITERATURE REVIEW}

Research on tumors with CSF has great focus nowadays due to their potential for analyzing former growth designs and morphological alterations in the cancer operation $[2,4,5]$. Early detection of a tumor in the brain is essential as the mortality rate is more prominent among human beings inducing brain tumors [2]. Brain tumor and CSF detection techniques employ image processing that has present for the past few decades. Several researchers have presented lots of automatic and semi-automatic image processing methodologies to detect the brain tumors in which most of the techniques fail to afford efficient and effective results owing to the presence of image noise, in uniformity, poor quality image contrast that takes place generally in medical images $[3,5,6]$. Scientists have applied different approaches to give build the best system that can detect a tumor from brain images in which MRI has the best image results. These approaches are proposed for MRI image segmentation, several statistical methods like to label pixels, using the threshold method, and parametric ones are widely employed [7-9]. These strategies name pixels as per likelihood values, which are resolved in light of the intensity distribution of the image.

They present the segmentation concept in Biomedical Engineering implication. Varying intensity of tumors in cerebrum magnetic resonance images (MRIs) makes the cerebrum of such tumors tremendously difficult. Cerebrum tumor division utilizing MRI has been an exceptional research range. Both elements based and chart book based techniques and also their blends have been proposed for cerebrum tumor division [2]. The authors state that brain segmentation is computerized utilizing the dual localization strategy. In the initial step of their procedure scull veil is produced for the MRI images. White matter and tumor area are utilized to ad-lib K-implies algorithm. In the last stride of their strategy, they evaluated the breadth and length [6]. MRI is valuable for analyzing cerebrum images as a result of its high precision rate. The proposed strategy joins the clustering and arrangement algorithm to minimize the error rate. Segmentation errand is performed utilizing orthonormal administrators and characterization utilizing BPN. Pictures having the tumor are prepared to utilize K-implies grouping and a critical precision rate of $75 \%$ is acquired [1].

The author [7], highlight that segmentation results won't be exact if the tumor edges are not sharp, and this case emerge amid the underlying phase of a tumor. The texture-based strategy is proposed in this paper. Alongside cerebrum tumor location, segmentation is likewise done automatically utilizing this technique.

The author proposed a proficient strategy for cerebrum tumor identification. A standout amongst the most critical strides in tumor discovery is segmentation. A mix of two standard algorithms, first means move and second standardized slice is performed to identify the mind tumor surface zone in MRI. By utilizing a mean move algorithm pre-handling step is performed with a specific end goal to shape fragmented districts. In the following stride district hubs clustering are prepared by the standardized cut strategy. In the last stride, the cerebrum tumor is distinguished through segment investigation.

The author [9] propose a programmed mind tumor location approach utilizing symmetry examination. They first distinguish a tumor, section it and afterwards discover the region of a tumor. One of the vital elements is that after playing out the quantitative examination, we can distinguish the status of an expansion in the ailment. They have recommended a multi-step and secluded drew closer to take care of the intricate MRI division issue. Tumor discovery is the initial step of tumor division. They have gotten great outcomes in complex circumstances. In this paper, we display another approach for computerized brain tumor discovery. The examination chiefly comprises of four stages which incorporate with pre-processing, isolation of an area utilizing crop tool, feature enhancing of the district by utilizing histogram and final 
grouping utilizing the support vector machine. In the pre-handling stage, channels are utilized to evacuate picture commotion introduce in the MRI image [7-9].

\section{METHODOLOGY}

The main objective of our work is to develop a system that can auto-detect the tumorous region and combination of CSF that can segregate between the tumorous and non-tumorous patient, initially the input MRI image is pre-processed to fit the image for the rest of the process. The research mainly consists of four steps which include pre-processing, segregation of a region using the crop tool, feature enhancing of the region by using HoG feature and final classification using the support vector machine as shown in Figure 2. Figure 3 shows the method of training data sets of MRI images and using the segmentation process of all extracted features which is a part of training and testing sets of database.

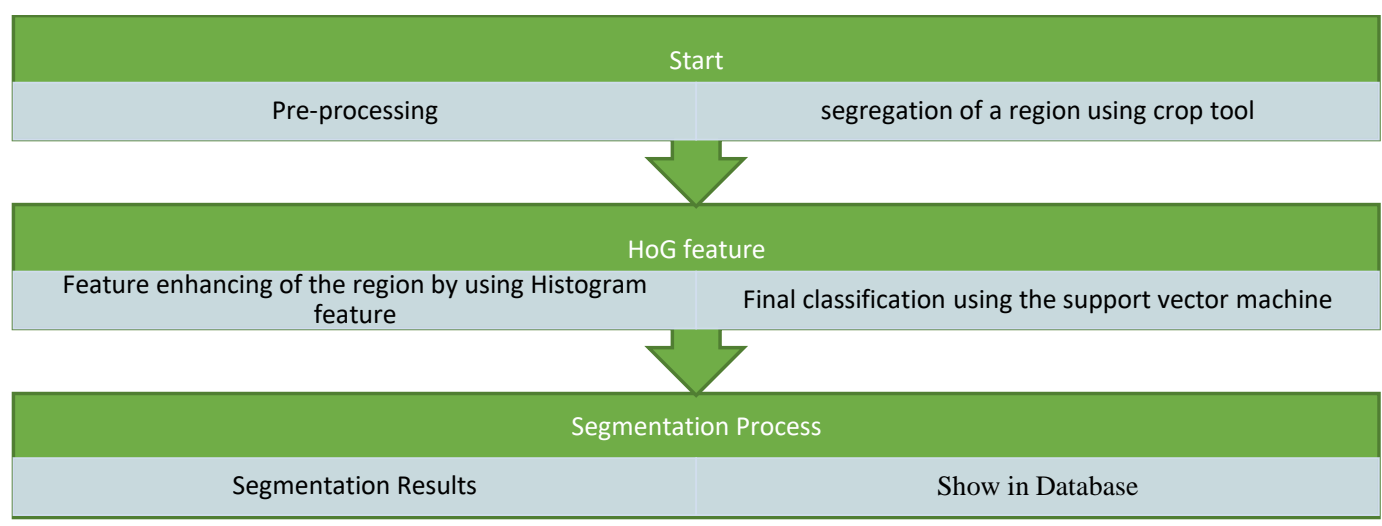

Figure 2. Proposed framework

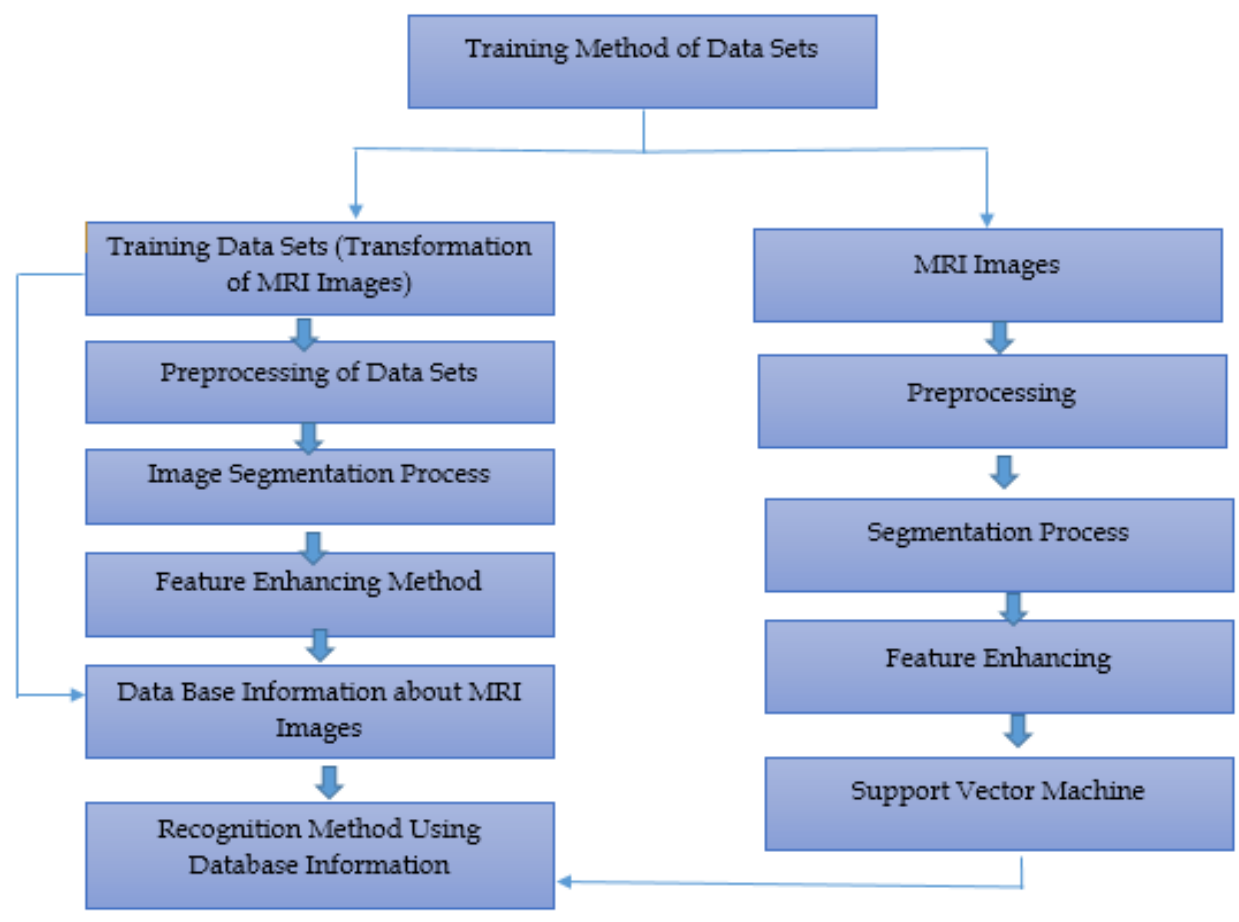

Figure 3. Proposed methodology of learning model [1] 


\section{PROPOSED TECHNIQUE FOR DETECTION OF TUMOR}

\subsection{Pre-processing}

Pre-processing are used to focus on accurate MRI image components. The investigator used dark MRI images so that the researcher smoothed it for better results by a change in contrast.

\subsection{Image enhancement}

Image enhancement is the digital image correction technique to make the images more suitable for further diagnosis. The Tumor research nowadays focuses incredibly on their potential to separate past progression arrangements and morphological changes in development, operation [10-12]. The recent work on the cerebral tumor, like CSF, is relevant as the rate of death among individuals acting on a brain tumor is more evident [13]. Techniques use the processing of images that have existed in recent decades. The researchers presented systems of modified and semi-automatic image processing approaches to detect tumors in the cerebral district in which most frameworks disregard the cost of capable and effective results due to the region of image noise, inconsistency and low-quality images generally found in medical images [14-16]. The researchers have linked obvious techniques with a common final objective to provide the best system capable of separating a tumor from a cerebral image with the best image results for MRI. Such techniques label pixels as shown by the values of probability calculated based on power propagation. Some of them are removing noise from your image, you can only imagine the brain cortex territory in MRI [10]. The specific dark ranges of the brain are excluded from the system improved by the image [17].

\subsection{Feature enhancing}

The researcher improves the histogram characteristics of selected regions, marking our area as positive and negative, and passing our vector to train our model. Feature enhancement is one of the most critical steps when distinguishing or separating a specific region from your image, numerous algorithms rely on the feature direction [17-19]. The researchers use the histogram feature to identify the element of interest in our location. Oriented gradient histogram can be used to recognize objects in an image. In general, as explained in the paper "Pedestrian Detection using Histogram of Oriented Gradients" by Dalal and Triggs are used for pedestrian detection. The researchers are trying to distinguish tumor area in the cortex of the brain [20-22]. Histogram, the position of objects is a process in which an image detector detects image characteristics and determines the proximity of objects of interest whether they occur in the image [23-25].

\section{RECORD OF DATASET}

The MRI images dataset that we have used in our work has been divided into two categories:

\subsection{Training dataset}

The training data set was obtained from Google sources to gather MRI images of tumors as well as CSF that the researcher used to train our proposed model. The researcher took several tumor MRI images for training.

\subsection{Testing dataset}

The researcher tested our model on a dataset collected in multiple locations from the multiple famous hospitals. As the hospital has the availability of only 100 patient's data record that is suffering from brain tumors with CSF symptoms. In Figure 4 shows that highlighted points of disease which indicate that the problem creating inside of the brain.
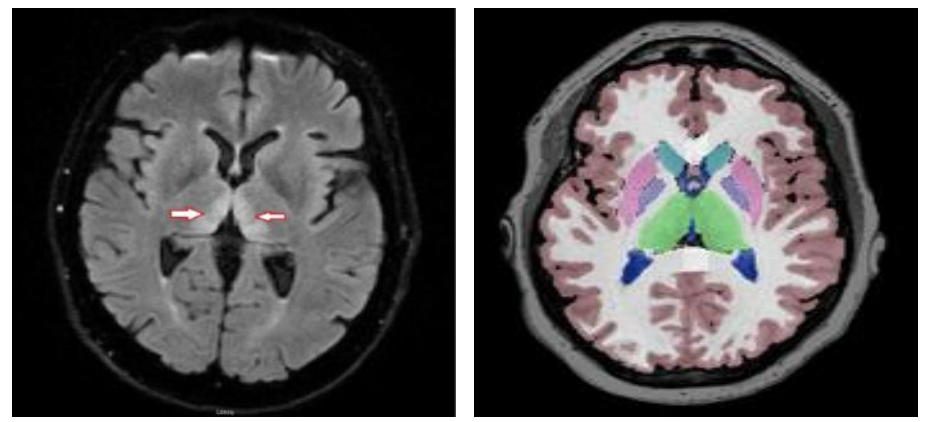

Figure 4. Training data set and testing data set of patients [1] 


\subsection{Classification}

In this area, to arrange the data image, whether the image contains tumorous region with CSF symptoms or not by utilizing support vector machine (SVM) classifier. SVMs are generally called Support vector networks that are observed in finding illustrations with related finding calculations, which examine the process.

\section{CONCLUSION}

The reason behind this study is to use a brain cancer location system through MRI brain images. The MRI data are obtained from the brain web database and shows a sample MRI brain image. In this research paper, the researchers are working on these images, which are based on tumors, applying segment region for tumor as well as CSF. These images represent the size of the tumor and need to calculate the size of the tumor with the help of MATLAB. In this research work, the author is focusing on the size of a tumor and calculate the area of the region from first to the last step until the growing sizes of a tumor cell. By using a different MATLAB tool. We have tested samples of frequency and also applied autocorrelation. Brain tumors are the main area of our research; accuracy is the main tool of success, which is why this study proposes MRI to get the best images and best results. This study depicts the brain tumor utilizing images division of brain tumor among the MRI pictures and demonstrates the outcomes by our recently proposed calculation. The reason for this study is to cerebrum tumor location systems through MRI brain images. This study explores the importance of pathologist as it is a bit hard for a common man to observe the tumor region easily just by viewing MRI films as pathologists and MRI technicians can judge the tumor area by doing a comparative analysis of an image. At that position, we connected characteristic extraction strategy by applying Histogram of gradient feature after which it worked on an assistance vector machine in request to assemble a classifier that could ready to predict tumorous and non-tumorous MRI examinees were trying to observe that; is our system able to detect the correct region that is also highlighted by a pathologist. All strategies are similarly useful for a specific kind of picture. In future, this software engineer should be possible more propelled with the goal that sort can arrange tumors.

\section{REFERENCES}

[1] S. Saeed et al., "Performance analysis the of cerebrospinal fluid (CSF) cell diseases with interfacing of MRI 4-dimensional segmentation," Images processing, pp.1-12, 2019.

[2] K. Usman and K. Rajpoot, "Brain tumor classification from multi-modality MRI using wavelets and machine learning," Pattern Analysis and Applications, vol. 20, no. 3, pp. 871-881, 2017.

[3] C. Desantis, R. Siegel, and A. Jemal, "Cancer treatment \& survivorship facts \& figures 2012-2013,"Atlanta, GA: American Cancer Society, 2012.

[4] L. A. Green et al., "A multicentre, prospective, randomized, controlled study to evaluate the use of a fibrin sealant as an adjunct to sutured dural repair," British journal of neurosurgery, vol. 29, no. 1, pp. 11-17, 2015.

[5] L. W. Schievink et al., "A classification system of spontaneous spinal CSF leaks," Neurology, vol. 87, no. 7, pp. 673-679, 2016.

[6] A. A. Badarnel, H. Nafadat, and A. M. Alraziqi, "A classifier to detect tumor disease in brain MRI brain images," ACM international conference on advances in social networks analysis and mining, Istambul, pp. 784-787, 2012.

[7] S. Giovanni et al., "Galeapericranium dural closure: can we safely avoid sealants," Clin. Neurol. Neurosurg., vol. 123, pp. 50-54, 2014.

[8] S. Saeed and Raza Jafri, "Estimation of brain tumor using latest technology of mobile phone," Journal of Information \& Communication Technology (JICT), vol. 9, no. 1, pp. 23-32, 2015.

[9] S. Saeed et al., "Combining Fourier and lagged k-nearest neighbour missing values imputation for brain cancer and CSF," Plose one, pp. 1-12, 2019.

[10] W. Wu, et al., "Brain tumor detection and segmentation in a CRF (conditional random fields) framework with pixel-pairwise affinity and superpixel-level features," International journal of computer assisted radiology and surgery, vol. 9, no. 2, pp. 241-253, 2014.

[11] I. Altaf, A. H. Vohra, and S. Shams. "Management of cerebrospinal fluid leak following posterior cranial fossa surgery," Pakistan Journal of Medical Sciences, vo. 32, no. 6, pp. 1439, 2016.

[12] D. R. Horn and B. Chen, "Light shop: Interactive light field manipulation and rendering," In Proc. Symposium on Interactive 3D Graphics and Games, Washington, vol. 19, pp. 1-18, 2007.

[13] T. Logeswari and M. Karnan, "An improved implementation of brain tumor detection using segmentation based on soft computing," Journal of Cancer Research and Experimental Oncology, vol. 2, no. 1, pp. 6-14, 2010.

[14] H. Selvaraj, S. Thamaraiselvi, D. Selvathi and L. Gewali, "Brain MRI slices classification using least squares support vector machine," International Journal of Intelligent Computing in Medical Sciences and Image Processing, vol. 1., no. 1, pp. 21-33, 2007.

[15] M. Sezgin and B. Sankar, "Survey over image thresholding techniques and quantitative performance evaluation," J. Electron imaging, vol. 13, no. 1, pp.146-165, 2004. 
[16] S. Soobia and A. Abdullah, "Investigation of a brain cancer with interfacing of 3-dimensional image processing," International Conference on Information Science and Communication Technology (ICISCT), pp. 1-6, 2019.

[17] S. Saeed, et al., "Implementation of fourier transformation with brain cancer and CSF images," Indian Journal of Science \& Technology, vol. 12, no. 37, pp. 1-9, 2019.

[18] S. Saeed et al., "Analysis of the lung cancer patient's for data mining tool," Indian Journal of Science \& Technology, 2019.

[19] S. Yatsushiro et al., "Correlation mapping for visualizing propagation of pulsatile CSF motion in intracranial space based on magnetic resonance phase contrast velocity images: preliminary results," 36th Annual International Conference of the IEEE Engineering in Medicine and Biology Society, pp. 3300-3303, 2014.

[20] A. Abdullah et al., "Cerebrospinal fluid pulsatile segmentation-a review," The 5th Biomedical Engineering International Conference, pp. 1-7, 2012.

[21] Z. Abd. Algfoor, M. S. Sunar, and A. Abdullah. "A new weighted pathfinding algorithm to reduce the search time on grid maps," Expert Systems with Applications, vol. 71, pp. 319-331, 2017.

[22] A. Abdullah et al., "An improved local best searching in particle swarm optimization using differential evolution," 11 th International Conference on Hybrid Intelligent Systems (HIS), pp. 115-120, 2011.

[23] A. Abdullah, et al., "An improved swarm optimization for parameter estimation and biological model selection," PloS one, vol. 8, no. 4, pp. 1-16, 2013.

[24] A. Gupta and G. Pahuja, "Hybrid clustering and boundary value refinement for tumor segmentation using brain MRI," IOP Conference Series: Materials Science and Engineering, vol. 225, no. 1, pp. 1-8, 2017.

[25] L. A. Van der Kleij et al., "Fast CSF MRI for brain segmentation; cross-validation by comparison with 3D T1-based brain segmentation methods," PLoS ONE, vol. 13, no. 4, pp 1-14 2018.

\section{BIOGRAPHIES OF AUTHORS}

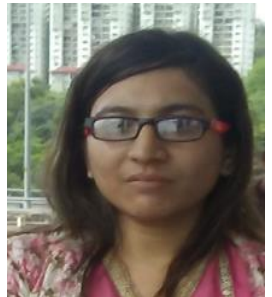

Ms Soobia Saeed is working as an Assistant Professor, Head of publication Department, and Coordinator of Seminars and Training at Institute of Business \& Technology-IBT, Karachi, Pakistan. Currently, she is a PhD Scholar in software engineering, from University Teknologi Malaysia-UTM, Malaysia She did MS in Software Engineering from Institute of Business \& Technology-IBT, Karachi, Pakistan, and Masters in Computer Science from Institute of Business \& Technology-IBT, Karachi, Pakistan and Bachelors in Mathematical Science from Federal Urdu University of Art, Science \& Technology (FUUAST), and Karachi, Pakistan. She is a farmer research Analytic from University Teknologi Malaysia and supervises ICT \& R and D funded Final Year Project (FYP).

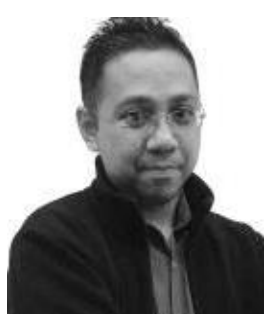

Dr Afnizanfaizal Abdullah is a senior lecturer at the School of Computing, with a $\mathrm{PhD}$. in Computer Science, specializing in artificial intelligence techniques for analyzing biological data. My research interests are in the designing of machine learning algorithms for healthcare applications in cloud environments. In 2015, I have co-founded Synthetic Biology Research Group to drive innovation in research and development of healthcare, biotechnology, and environment areas through computing and engineering. I am also active in engaging with industrial partners and professional communities to contribute the knowledge and skills for the public. 